International Journal of Mathematics for Industry

Vol. 11, No. 1 (2019) 1950002 (10 pages)

(C) The Author(s)

DOI: $10.1142 /$ S2661335219500023

\title{
Predicting precision matrices for color matching problem
}

\author{
Takayoshi Nakamoto*, ${ }^{*}$, Ryuei Nishii ${ }^{\dagger}$ and Shinto Eguchi* \\ *Department of Vehicle Development \\ Mazda Motor Corp., 3-1 Shinchi, Fuchu-cho \\ Aki-gun, Hiroshima 730-86\%0, Japan \\ $\dagger$ Institute of Mathematics for Industry \\ Kyushu University, 744 Motooka \\ Nishi-ku, Fukuoka 819-0385, Japan \\ $\$$ The Institute of Statistical Mathematics \\ 10-3 Midori-cho, Tachikawa, Tokyo 190-8562, Japan \\ §nakamoto.tak@mazda.co.jp
}

Received 17 October 2018

Revised 9 January 2019

Accepted 17 January 2019

Published 28 May 2019

\begin{abstract}
In this paper, as data, ellipsoids in a color coordinate called the Commission Internationale de l'Eclairage (CIE)-Lab system are given as data for 19 colors. Each ellipsoid is a region where all points are visually recognized as the same color at the center of the coordinate system. Our aim here is to predict the shape of an ellipsoid whose center is given by a new color. We proposed two prediction methods of positive definite matrices determining ellipsoids.

The first one is a nonparametric method with Gaussian kernel. The prediction is provided as a weighted sum of positive definite matrices corresponding to 19 ellipsoids in the training data. The second one is to use a matrix-valued regression model applied to a logarithm of positive definite matrices where explanatory variables are three elements of color centers. The best result was obtained by the nonparametric methods with three bandwidth parameters. The log normal regression had a weaker performance, but even so the model estimation was easily carried out.
\end{abstract}

Keywords: Color matching problem; kernel regression; log normal regression; nonparametric estimation; positive definite matrix; Riemannian metric.

\section{Introduction}

When car motor companies develop a new color or texture, it is very important to ensure color matching between the various products or parts in order to provide a sophisticated appearance and design. First, we briefly review the color coordinate systems here.

One example of a color coordinate system is the Commission Internationale de l'Eclairage (CIE)

This is an Open Access article published by World Scientific Publishing Company. It is distributed under the Creative Commons Attribution 4.0 (CC BY) License. Further distribution of this work is permitted, provided the original work is properly cited. 
Lab system. ${ }^{1}$ Each color in this system is represented by $L$ (lightness) and $a$ for the green-red and $b$ for the blue-yellow color components. ${ }^{2}$ An example is shown in Fig. 1(a), where $L$ takes a value from 0 to 100 and $a$ and $b$ range from $-90^{\circ}$ to $90^{\circ}$.

The human visual system has a dead zone characteristic in that a set of colors with little difference is visually recognized as the same color. ${ }^{3}$ This dead zone is represented by an ellipsoid in the Lab space with the color center of interest. ${ }^{5}$ Therefore, predicting a dead zone ellipsoid corresponding to a new color is required in the Lab space in order to know the acceptable zone. This is known as the color matching problem.

The DuPont dataset ${ }^{6}$ gives 19 pairs of colors and ellipsoids in the Lab space. See Fig. 1(b) for color centers and corresponding ellipsoids. Note that the shapes of the ellipsoids are different from color to color. Moreover, similar colors have similar shapes in Lab space. ${ }^{7}$ For example, "Strong orange yellow" and "Moderate yellow" have similar shapes (long and thin for the $b$ coordinate), and "Moderate green", "Moderate bluish green", and "Dark bluish green" have similar shapes (long and thin for the $a$ coordinate).

The dataset is represented as $\left(\mathbf{u}_{i}, \mathbf{G}_{i}\right)$ for $i=1,2, \ldots, 19$, where $\mathbf{u}_{i}=\left(a_{i}, b_{i}, L_{i}\right)^{T}$ denote color centers and $\mathbf{G}_{i}=\left(g_{i j k}\right), j, k=1,2,3$ denote precision matrices (positive definite matrices) giving ellipsoids for colors $\mathbf{u}_{i}$. The dataset will be given in Sec. 2.
The problem considered here is how to predict an ellipsoid or a positive definite matrix for a new color center given as $\mathbf{u}_{0}=\left(a_{0}, b_{0}, L_{0}\right)^{T} \in[-90,90]^{2} \times$ $[0,100]$. Then, the tolerance ellipsoid for the color with center $\mathbf{u}_{0}$ is given by an ellipsoid:

$$
\left(\mathbf{v}-\mathbf{u}_{0}\right)^{\mathrm{T}} \mathbf{G}\left(\mathbf{u}_{0}\right)\left(\mathbf{v}-\mathbf{u}_{0}\right) \leq 1,
$$

where $\mathbf{v}=(a, b, L)^{T}$ is a vector of variables in the Lab space, and

$$
\mathbf{G}\left(\mathbf{u}_{\mathbf{0}}\right)=\left(\begin{array}{lll}
g_{11}\left(\mathbf{u}_{\mathbf{0}}\right) & g_{12}\left(\mathbf{u}_{0}\right) & g_{13}\left(\mathbf{u}_{0}\right) \\
g_{12}\left(\mathbf{u}_{\mathbf{0}}\right) & g_{22}\left(\mathbf{u}_{0}\right) & g_{23}\left(\mathbf{u}_{0}\right) \\
g_{13}\left(\mathbf{u}_{\mathbf{0}}\right) & g_{23}\left(\mathbf{u}_{0}\right) & g_{33}\left(\mathbf{u}_{0}\right)
\end{array}\right),
$$

is a positive definite matrix corresponding to the color with center $\mathbf{u}_{0}$. We will estimate $\mathbf{G}\left(\mathbf{u}_{0}\right)$ through nonparametric and parametric methods in Secs. 2 and 3, respectively.

We consider three metrics between positive definite matrices as the accuracy assessment of the estimated ellipsoids. We also consider three normalizing methods for Gaussian weights to the observed positive definite matrices. To this end, we developed a nonparametric method of NadarayaWatson type estimation based on Gaussian kernel with several bandwidth parameters. Furthermore, we propose a matrix-valued regression to logarithm of positive definite matrices as response variables and three elements of color centers as explanatory variables.

The best result was obtained by the nonparametric methods with three bandwidth parameters. The log normal regression showed weaker

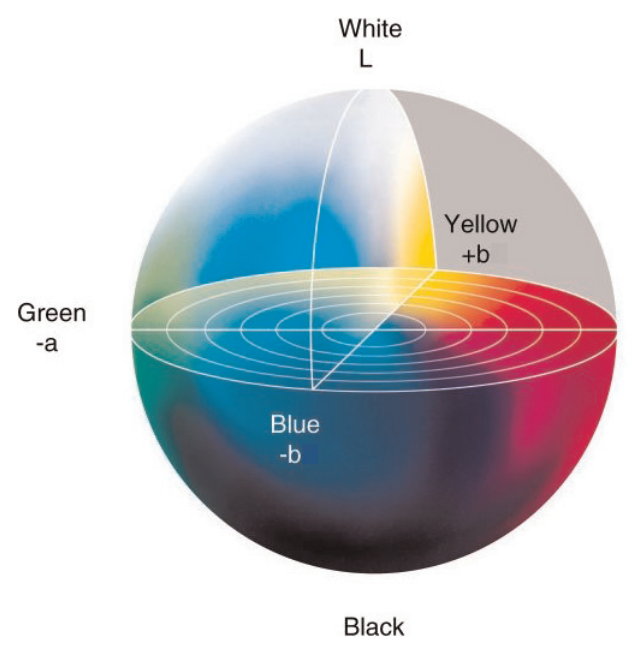

(a) CIE Lab color coordinate system

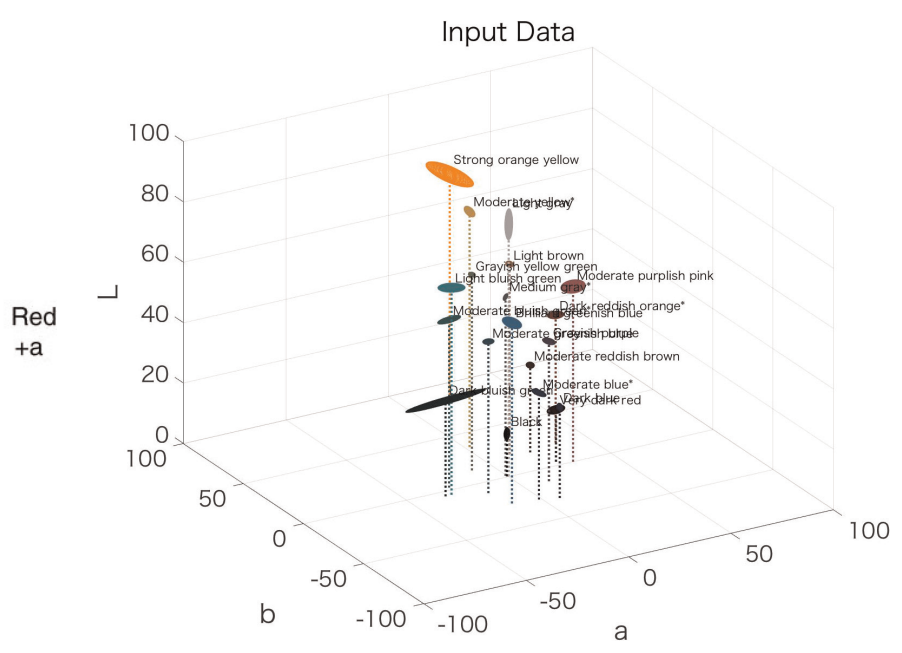

(b) Ellipsoids for DuPont data set

Fig. 1. CIE Lab color coordinate system and ellipsoid shapes. 
performance, but even so, its model estimation could easily be carried out. Both of the proposed methods can greatly reduce the cost of color matching compared with the conventional cut-andtry method, which would make the development of new colors more efficient.

\section{Nonparametric Prediction of Positive Definite Matrices}

Ellipsoid (1) of color $\mathbf{u}_{i}=\left(a_{i}, b_{i}, L_{i}\right)^{T}(i=1,2, \ldots$, 19) in the DuPont dataset is rewritten as

$$
\left(\mathbf{v}-\mathbf{u}_{i}\right)^{\mathrm{T}} \mathbf{V}_{i}^{-1}\left(\mathbf{v}-\mathbf{u}_{i}\right) \leq 1
$$

where $\mathbf{v}=(a, b, L)^{T}$ and

$$
\mathbf{V}_{i}=\left(\begin{array}{lll}
v_{i 11} & v_{i 12} & v_{i 13} \\
v_{i 12} & v_{i 22} & v_{i 23} \\
v_{i 13} & v_{i 23} & v_{i 33}
\end{array}\right),
$$

is a positive definite matrix corresponding to the variance-covariance matrix. Table 1 lists the color names, centers $\mathbf{u}_{i}$, and matrices $\mathbf{V}_{i}$ of the DuPont dataset.

\subsection{Normalization of input vectors}

Hereafter, color centers $\mathbf{u}_{i}=\left(a_{i}, b_{i}, L_{i}\right)^{T}$ are used for the expression of $\mathbf{V}_{i}$. Table 2 summarizes the basic statistics of Table 1. It is clear here that the sample means are significantly different. Therefore, we consider the following normalization methods for deriving Gaussian kernel weights.

\subsubsection{First normalization method}

Let $\mathbf{u}=(a, b, L)^{T} \in[-90,90]^{2} \times[0,100]$ denote a color in Lab space. Then, we define the following normalization:

$$
\psi_{1}(\mathbf{u})=\left(\frac{a}{90}, \frac{b}{90}, \frac{L}{50}-1\right) \in[-1,1]^{3},
$$

where we take denominators 90 or 50 so that the transformed values belong to an interval $[-1,1]$.

\subsubsection{Second normalization method}

The second normalization is based on sample means and standard deviations.

$$
\psi_{2}(\mathbf{u})=\left(\frac{a-\bar{a}}{s_{a}}, \frac{b-\bar{b}}{s_{b}}, \frac{L-\bar{L}}{s_{L}}\right) \in \mathbb{R}^{3},
$$

where $\bar{a}$ and $s_{a}^{2}$ denote the sample means and the sample variance of $a_{i}, \bar{b}, s_{b}^{2}, \bar{L}$, and $s_{L}^{2}$ are similarly defined.

\subsubsection{Third normalization method}

The third normalization is to transform $\mathbf{u}$ into the unit cube as

$$
\begin{aligned}
\psi_{3}(\mathbf{u}) & =\left(\frac{a-a_{\min }}{a_{\max }-a_{\min }}, \frac{b-b_{\min }}{b_{\max }-b_{\min }}, \frac{L-L_{\min }}{L_{\max }-L_{\min }}\right) \\
& \in[0,1]^{3}
\end{aligned}
$$

where $a_{\min }=\min \left\{a_{1}, \ldots, a_{n}\right\}$. Similarly, $a_{\max }, b_{\min }$, $b_{\max }, L_{\min }$ and $L_{\max }$ are defined.

Table 1. Color names, centers and ellipsoids from the DuPont dataset.

\begin{tabular}{|c|c|c|c|c|c|c|c|c|c|c|}
\hline $\mathrm{i}$ & Center Names & $a$ & $b$ & $L$ & $v_{11}$ & $v_{22}$ & $v_{12}$ & $v_{13}$ & $v_{23}$ & $v_{33}$ \\
\hline 1 & Moderate blue & -1.403 & -27.81 & 35.338 & 1.784 & 5.015 & -1.166 & -0.053 & 0.268 & 0.850 \\
\hline 2 & Moderate greenish blue & -16.374 & -11.263 & 50.259 & 2.564 & 2.340 & 0.118 & -0.065 & -0.030 & 0.618 \\
\hline 3 & Medium gray & -0.782 & 1.049 & 59.334 & 0.779 & 1.955 & -0.131 & 0.033 & 0.134 & 0.777 \\
\hline 4 & Moderate bluish green & -27.549 & 2.374 & 55.618 & 5.892 & 1.752 & -0.362 & -0.300 & 0.154 & 0.950 \\
\hline 5 & Light brown & 12.606 & 20.571 & 62.928 & 1.735 & 2.302 & 0.369 & 0.024 & 0.145 & 0.878 \\
\hline 6 & Grayish purple & 12.153 & -13.079 & 46.389 & 2.109 & 3.725 & -0.962 & 0.030 & -0.084 & 0.810 \\
\hline 7 & Dark reddish orange & 35.646 & 21.403 & 42.315 & 3.661 & 2.801 & 0.587 & -0.093 & 0.114 & 1.039 \\
\hline 8 & Moderate yellow & 1.937 & 35.638 & 78.023 & 1.645 & 4.131 & 0.249 & -0.020 & 0.214 & 1.285 \\
\hline 9 & Grayish yellow green & -10.011 & 13.281 & 64.938 & 1.433 & 2.357 & -0.277 & -0.144 & 0.135 & 0.762 \\
\hline 10 & Black & -0.453 & 0.421 & 14.14 & 0.627 & 2.096 & -0.064 & 0.007 & -0.057 & 1.520 \\
\hline 11 & Light bluish green & -30.732 & -5.03 & 68.678 & 6.132 & 4.070 & -1.127 & -0.418 & 0.108 & 1.091 \\
\hline 12 & Moderate reddish brown & 21.121 & 17.804 & 28.893 & 1.243 & 2.630 & 0.411 & -0.093 & -0.199 & 1.027 \\
\hline 13 & Dark bluish green & -33.638 & -5.012 & 31.683 & 21.938 & 1.522 & 1.586 & -1.519 & 0.019 & 1.267 \\
\hline 14 & Brilliant greenish blue & -13.44 & -25.897 & 59.904 & 3.119 & 5.599 & -1.249 & -0.042 & -0.538 & 1.299 \\
\hline 15 & Very dark red & 25.237 & 3.409 & 17.357 & 4.261 & 2.691 & -0.182 & -0.262 & 0.264 & 1.248 \\
\hline 16 & Moderate purplish pink & 31.509 & -0.183 & 58.109 & 5.831 & 3.988 & -0.422 & 0.080 & -0.123 & 1.742 \\
\hline 17 & Dark blue & 6.826 & -31.146 & 30.186 & 1.769 & 3.030 & -1.096 & -0.006 & -0.011 & 1.074 \\
\hline 18 & Light gray & 0.307 & 0.214 & 83.481 & 1.099 & 5.275 & -0.507 & 0.146 & -0.100 & 1.899 \\
\hline 19 & Strong orange yellow & 18.226 & 79.894 & 76.057 & 3.468 & 19.339 & -0.025 & 0.187 & 0.657 & 2.398 \\
\hline
\end{tabular}


Table 2. Basic statistics of color centers.

Sample means Standard deviations Minimum Maximum

\begin{tabular}{lrrrr}
\hline$a_{i}$ & 1.641 & 20.236 & -33.638 & 35.646 \\
$b_{i}$ & 4.034 & 25.276 & -31.146 & 79.894 \\
$L_{i}$ & 50.717 & 20.289 & 14.140 & 83.481 \\
\hline
\end{tabular}

\subsection{Metrics between positive definite matrices}

Let $\mathbf{L}=\left(l_{j k}\right)$ and $\mathbf{M}=\left(m_{j k}\right)$ be positive definite matrices of order 3 . We use the following three metrics for evaluating the positive definite matrices in order to obtain the best Gaussian kernel estimations.

\subsubsection{Frobenius norm}

The Frobenius norm gives the Euclidean norm between two matrices.

$$
\Delta_{F}(\mathbf{L}, \mathbf{M})=\|\mathbf{L}-\mathbf{M}\|_{F}=\sqrt{\sum_{j=1}^{3} \sum_{k=1}^{3}\left(l_{j k}-m_{j k}\right)^{2}} .
$$

\subsubsection{Riemannian metric}

The Riemannian metric is obtained from the geometrical distance between two matrices. ${ }^{8}$

$$
\Delta_{R}(\mathbf{L}, \mathbf{M})=\sqrt{\sum_{j=1}^{3}\left\{\log \lambda_{j}\left(\mathbf{L}^{-1} \mathbf{M}\right)\right\}^{2}},
$$

where $\lambda_{j}\left(\mathbf{L}^{-1} \mathbf{M}\right)>0$ denotes the $j$ th largest eigenvalue of $\mathbf{L}^{-1} \mathbf{M}$.

\subsubsection{Kullback-Leibler (KL) divergence}

We apply the Kullback-Leibler (KL) divergence between $N(\mathbf{0}, \mathbf{L})$ and $N(\mathbf{0}, \mathbf{M})$ by regarding two matrices as the variance-covariance matrices of two independent Gaussian distributions having zero mean, as

$$
\Delta_{K L}(\mathbf{L}, \mathbf{M})=\frac{1}{2}\left\{\log \left|\mathbf{L}^{-1} \mathbf{M}\right|+\operatorname{trace}\left(\mathbf{L}^{-1} \mathbf{M}\right)-3\right\} .
$$

The first two metrics satisfy the three axioms of the distance whereas KL does not.

\subsection{Nonparametric estimation of a center matrix by Gaussian kernel}

Gaussian kernel estimation is applied to the positive definite matrices. ${ }^{9}$ We set $\mathcal{V}$ as the space containing all positive definite matrices of order $p$. Assume that training dataset $\left\{\mathbf{V}_{i} \in \mathcal{V} \mid i=1, \ldots, n\right\}$ is available. Then, our aim here is to derive an ellipsoid i.e., a matrix $\mathbf{V} \in \mathcal{V}$ of the training data in some sense. The matrix is defined by minimizing the following loss function:

$$
\begin{aligned}
L(\mathbf{V})= & \sum_{i=1}^{n} w_{i} \Delta_{K L}\left(\mathbf{V}, \mathbf{V}_{i}\right) \\
& \text { for weights } w_{i}>0 \text { with } \sum_{i} w_{i}=1,
\end{aligned}
$$

where $\Delta_{\mathrm{KL}}(\cdot, \cdot)$ is the KL divergence defined in (10).

\section{Proposition.}

Let

$$
\hat{\mathbf{V}}=\sum_{i=1}^{n} w_{i} \mathbf{V}_{i} \in \mathcal{V},
$$

be a weighted sum of the training data. Then, it holds that

$$
\hat{\mathbf{V}}=\arg \min _{\mathbf{V} \in \mathcal{V}} L(\mathbf{V}) .
$$

Proof. We obtain that

$$
2 L(\hat{\mathbf{V}})=\sum_{i} w_{i}\left[\operatorname{trace}\left(\mathbf{V}_{i} \hat{\mathbf{V}}^{-1}\right)-p+\log \left|\mathbf{V}_{i} \hat{\mathbf{V}}^{-1}\right|\right] .
$$

By the linearity of the trace function, we have

$$
\begin{aligned}
2 L(\mathbf{V}) & -2 L(\hat{\mathbf{V}}) \\
= & \operatorname{trace}\left(\mathbf{V}^{-1} \hat{\mathbf{V}}\right)-\log |\mathbf{V}|-p+\log |\hat{\mathbf{V}}| \\
= & \operatorname{trace}\left(\mathbf{V}^{-1} \hat{\mathbf{V}}\right)-p+\log \left|\mathbf{V}^{-1} \hat{\mathbf{V}}\right| \geq 0 .
\end{aligned}
$$

The last inequality follows from the non-negativity for KL divergence (10). Note that the equality holds if and only if $\mathbf{V}=\hat{\mathbf{V}}$.

The proposition implies that the weighted sum (12) minimizes the loss function (11). Next, determination methods of the weights $w_{i}$ are proposed from different viewpoints.

\subsubsection{Gaussian kernel estimation 1}

In the first method for predicting the positive definite matrix for a certain color center $\mathbf{u}$, we 
normalize input vector $\mathbf{u}$ and obtain $\psi_{q}(\mathbf{u})$ defined by (5)-(7) for $q=1,2,3$. Next, we apply a single bandwidth tuning parameter $\gamma>0$ to the nonlinear weighting function:

$$
\begin{gathered}
\hat{\mathbf{V}}\left(\psi_{q}(\mathbf{u})\right)=\sum_{i=1}^{n} w_{i}\left(\psi_{q}(\mathbf{u}), \gamma\right) \mathbf{V}_{i}, \\
w_{i}\left(\psi_{q}(\mathbf{u}), \gamma\right)=\frac{\exp \left\{-\gamma\left\|\psi_{q}(\mathbf{u})-\psi_{q}\left(\mathbf{u}_{i}\right)\right\|^{2}\right\}}{\sum_{l=1}^{n} \exp \left\{-\gamma\left\|\psi_{q}(\mathbf{u})-\psi_{q}\left(\mathbf{u}_{l}\right)\right\|^{2}\right\}} .
\end{gathered}
$$

If $\gamma=0,(2.12)$ gives the mean matrix of $\mathbf{V}_{i}$. The positive definiteness of the matrix given by (14) is easily shown because it is given by the sum of positive weights on positive definite matrices.

The validity of estimation using the weighted sum in the form of (15) is ensured by the proposition. The bandwidth parameter $\gamma>0$ is tuned by minimizing a metric between the estimated and observed positive definite matrices using leave-oneout cross-validation. We consider three types of metrics: the Frobenius norm, the Riemannian metric, and the KL divergence.

Let $\hat{\mathbf{V}}_{i}$ be the estimated positive definite matrix in the form of (14) with (15) using the training data while omitting $\mathbf{V}_{i}$. We tune parameter $\gamma$ using the three metrics.

\section{Frobenius norm}

$$
\hat{\gamma}_{F}=\arg \min _{\gamma>0} \sum_{i=1}^{n} \Delta_{F}^{2}\left(\mathbf{V}_{i}, \hat{\mathbf{V}}_{i}\right),
$$

where $\Delta_{F}(\cdot, \cdot)$ denotes the Frobenius norm (8).

The tuning methods using the Riemannian metric and KL divergence are used similarly. Then, $\hat{\gamma}_{R}$ and $\hat{\gamma}_{K L}$ are similarly defined.

\subsubsection{Gaussian kernel estimation 2}

The diagonal elements in positive definite matrix $\mathbf{V}$ are more important than the off-diagonal elements because these parameters determine the ellipsoidal size corresponding to the color coordinates. We thus deconstruct the positive definite matrix $\mathbf{V}\left(\psi_{q}(\mathbf{u})\right)$ to the standard deviations and correlations as

$$
\mathbf{V}\left(\psi_{q}(\mathbf{u})\right)=\mathbf{S}\left(\psi_{q}(\mathbf{u})\right) \mathbf{C}\left(\psi_{q}(\mathbf{u})\right) \mathbf{S}\left(\psi_{q}(\mathbf{u})\right),
$$

where

$$
\begin{aligned}
& \mathbf{S}\left(\psi_{q}(\mathbf{u})\right) \\
& =\left(\begin{array}{ccc}
\sqrt{v_{11}\left(\psi_{q}(\mathbf{u})\right)} & 0 & 0 \\
0 & \sqrt{v_{22}\left(\psi_{q}(\mathbf{u})\right)} & 0 \\
0 & 0 & \sqrt{v_{33}\left(\psi_{q}(\mathbf{u})\right)}
\end{array}\right), \\
& \mathbf{C}\left(\psi_{q}(\mathbf{u})\right)=\left(\begin{array}{ccc}
1 & \rho_{12}\left(\psi_{q}(\mathbf{u})\right) & \rho_{13}\left(\psi_{q}(\mathbf{u})\right) \\
\rho_{12}\left(\psi_{q}(\mathbf{u})\right) & 1 & \rho_{23}\left(\psi_{q}(\mathbf{u})\right) \\
\rho_{13}\left(\psi_{q}(\mathbf{u})\right) & \rho_{23}\left(\psi_{q}(\mathbf{u})\right) & 1
\end{array}\right) .
\end{aligned}
$$

The standard deviations and correlations are estimated using different bandwidths $\gamma>0$ and $\delta>0$ as

$$
\begin{aligned}
\sqrt{v_{j j}\left(\psi_{q}(\mathbf{u})\right)}= & \sum_{i=1}^{n} w_{i}\left(\psi_{q}(\mathbf{u}), \gamma\right) \sqrt{v_{i j j}}, \quad j=1,2,3, \\
\rho_{j k}\left(\psi_{q}(\mathbf{u})\right)= & \sum_{i=1}^{n} w_{i}\left(\psi_{q}(\mathbf{u}), \delta\right) \rho_{i j k}, \\
& (j, k)=(1,2),(1,3),(2,3), \quad
\end{aligned}
$$

and $w_{i}\left(\psi_{q}(\mathbf{u}), \cdot\right)$ is defined by $(2.13)$. The $\rho_{i j k}$ is the $(j, k)$ correlation of matrix $\mathbf{V}_{i}$. Two bandwidths are estimated by minimizing the metric as

\section{Frobenius norm}

$$
\left(\hat{\gamma}_{F}, \hat{\delta}_{F}\right)=\underset{\gamma>0, \delta>0}{\operatorname{argmin}} \sum_{i=1}^{n} \Delta_{F}^{2}\left(\mathbf{V}_{i}, \hat{\mathbf{V}}_{i}\right),
$$

where $\hat{\mathbf{V}}_{i}$ is derived using (17) with two parameters.

Similarly, bandwidths tuned by the Riemannian metric and KL divergence are defined. The estimated matrix is shown to be positive definite because its correlation matrix is given by the sum of positive weights of the correlation matrices $\left(\rho_{i j k}\right)$.

Note that the tuned parameters depend on the normalization methods $\psi_{q}(\cdot)$. Note also that $\hat{\mathbf{V}}_{i}$ is positive definite because its correlation matrix is given by a linear combination of training correlation matrices with positive weights.

\subsubsection{Gaussian kernel estimation 3}

The first and second estimation methods in 2.3.1 and 2.3.2 use the common parameter $\gamma$ for the variances $v_{11}, v_{22}$ and $v_{33}$ for normalization types 
$q=1,2,3$. Actually, the maximum and the minimum of $v_{i 11}$ is given by 1.600 and 0.054 , respectively (see Table 1 ). This means that $v_{11}$ corresponding to color coordinate $a$ and the other two variances should be modeled separately. Under this consideration, we will propose the following third model:

$$
\begin{gathered}
\sqrt{v_{11}\left(\psi_{q}(\mathbf{u})\right)}=\sum_{i=1}^{n} w_{i}\left(\psi_{q}(\mathbf{u}), \gamma\right) \sqrt{v_{i 11}}, \\
\sqrt{v_{j j}\left(\psi_{q}(\mathbf{u})\right)}=\sum_{i=1}^{n} w_{i}\left(\psi_{q}(\mathbf{u}), \delta\right) \sqrt{v_{i j j}}, \quad j=2,3 \\
\rho_{j k}\left(\psi_{q}(\mathbf{u})\right)=\sum_{i=1}^{n} w_{i}\left(\psi_{q}(\mathbf{u}), \eta\right) \rho_{i j k} \\
(j, k)=(1,2),(1,3),(2,3)
\end{gathered}
$$

Then, the three bandwidths are tuned as follows.

\section{Frobenius norm}

$$
\left(\hat{\gamma}_{F}, \hat{\delta}_{F}, \hat{\eta}_{F}\right)=\arg \min _{\gamma>0, \delta>0, \eta>0} \sum_{i=1}^{n} \Delta_{F}^{2}\left(\mathbf{V}_{i}, \hat{\mathbf{V}}_{i}\right),
$$

where $\hat{\mathbf{V}}_{i}$ is estimated using (17) with three bandwidths.

Similarly, three bandwidths based on the Riemannian metric and $\mathrm{KL}$ divergence are respectively defined. Note that $\hat{\mathbf{V}}_{i}$ is positive definite for the same reason stated in the two-bandwidth case.

\section{Regression Analysis in Matrix Log-Normal Distribution}

Next, we construct a regression model between the positive definite matrices and color centers. ${ }^{11}$ Recall that $\left\{\left(\mathbf{u}_{i}, \mathbf{V}_{i}\right) \in \mathbb{R}^{3} \times \mathcal{V} \mid i=1, \ldots, n\right\}$ is the DuPont dataset with a set of positive definite matrices $\mathcal{V}$. In general, $\mathbf{V} \in \mathcal{V}$ has the following decomposition based on an orthogonal matrix $\mathbf{Q}$ as follows:

$$
\begin{gathered}
\mathbf{V}=\mathbf{Q D Q}^{T}, \\
\mathbf{D}=\left(\begin{array}{ccc}
\lambda_{1} & & \mathbf{0} \\
& \lambda_{2} & \\
\mathbf{0} & & \lambda_{3}
\end{array}\right), \quad \lambda_{i}>0 .
\end{gathered}
$$

Then, we define a logarithm of $\mathbf{V}$ by

$$
\log \mathbf{V}=\mathbf{Q}\left(\begin{array}{ccc}
\log \lambda_{1} & & \mathbf{0} \\
& \log \lambda_{2} & \\
\mathbf{0} & & \log \lambda_{3}
\end{array}\right) \mathbf{Q}^{T}
$$

Now, we use the log normal regression model ${ }^{12}$ described by

$$
\begin{array}{r}
\log \mathbf{V}_{i}:=\mathbf{M}_{0}+a_{i} \mathbf{M}_{1}+b_{i} \mathbf{M}_{2}+L_{i} \mathbf{M}_{3}+\mathbf{E}_{i}, \\
i=1,2, \ldots, n,
\end{array}
$$

where $\mathbf{M}_{j}(j=0,1,2,3)$ are unknown symmetric matrices of order 3 and $n=19$. They are regression coefficient matrices for explanatory variables $1, a_{i}, b_{i}$, and $L_{i}$. We assume that error matrices $\mathbf{E}_{i}(i=$ $1,2, \ldots, n)$ are distributed independently.

Let $\tilde{\mathbf{H}}=\left(\mathbf{H}_{1}, \ldots, \mathbf{H}_{n}\right)^{T}: 3 n \times 3 \quad$ with $\quad \mathbf{H}_{i}=$ $\log \mathbf{V}_{i}$, and $\tilde{\mathbf{M}}=\left(\mathbf{M}_{0}, \ldots, \mathbf{M}_{3}\right)^{T}: 12 \times 3$. The design matrix $\mathbf{X}$ is set as follows:

$$
\mathbf{X}=\left(\begin{array}{cccc}
1 & a_{1} & b_{1} & L_{1} \\
1 & a_{2} & b_{2} & L_{2} \\
\vdots & \vdots & \vdots & \vdots \\
1 & a_{n} & b_{n} & L_{n}
\end{array}\right): n \times 4
$$

Then, the log normal regression model (30) is rewritten as

$$
\mathbf{H}_{i}=\mathbf{M}_{0}+a_{i} \mathbf{M}_{1}+b_{i} \mathbf{M}_{2}+L_{i} \mathbf{M}_{3}+\mathbf{E}_{i},
$$

for $i=1,2, \ldots, n$, and we have the following simultaneous equation:

$$
\begin{aligned}
\tilde{\mathbf{H}} \equiv\left(\begin{array}{c}
\mathbf{H}_{1} \\
\mathbf{H}_{2} \\
\vdots \\
\mathbf{H}_{n}
\end{array}\right) \\
=\left(\begin{array}{cccc}
\mathbf{I}, & a_{1} \mathbf{I}, & b_{1} \mathbf{I}, & L_{1} \mathbf{I} \\
\mathbf{I}, & a_{2} \mathbf{I}, & b_{2} \mathbf{I}, & L_{2} \mathbf{I} \\
\vdots & \vdots & \vdots & \vdots \\
\mathbf{I}, & a_{n} \mathbf{I}, & b_{n} \mathbf{I}, & L_{n} \mathbf{I}
\end{array}\right)\left(\begin{array}{l}
\mathbf{M}_{0} \\
\mathbf{M}_{1} \\
\mathbf{M}_{2} \\
\mathbf{M}_{3}
\end{array}\right)+\left(\begin{array}{c}
\mathbf{E}_{1} \\
\mathbf{E}_{2} \\
\vdots \\
\mathbf{E}_{n}
\end{array}\right) \\
\\
\end{aligned}
$$

The regression coefficient matrix $\tilde{\mathbf{M}}$ is estimated by the least square method by minimizing the following error function:

$$
\begin{aligned}
\operatorname{trace}\left(\mathbf{S}^{T} \mathbf{S}\right)= & \operatorname{trace}\left(\tilde{\mathbf{M}}^{T} \tilde{\mathbf{X}}^{T} \tilde{\mathbf{X}} \tilde{\mathbf{M}}\right) \\
& -2 \operatorname{trace}\left(\tilde{\mathbf{M}}^{T} \tilde{\mathbf{X}}^{T} \tilde{\mathbf{H}}\right)+\operatorname{trace}\left(\tilde{\mathbf{H}}^{T} \tilde{\mathbf{H}}\right),
\end{aligned}
$$

where

$$
\mathbf{S}=\tilde{\mathbf{H}}-\tilde{\mathbf{X}} \tilde{\mathbf{M}}
$$


The derivative of the error function is defined as follows.

$$
\frac{\partial \operatorname{trace}\left(\mathbf{S}^{T} \mathbf{S}\right)}{\partial \tilde{\mathbf{M}}}=2 \tilde{\mathbf{X}}^{T} \tilde{\mathbf{X}} \tilde{\mathbf{M}}-2 \tilde{\mathbf{X}}^{T} \tilde{\mathbf{H}}=O .
$$

Then, the regression coefficient matrix is estimated as

$$
\begin{aligned}
\widehat{\tilde{\mathbf{M}}} & =\left\{\left(\mathbf{X}^{T} \mathbf{X}\right)^{-1} \otimes \mathbf{I}\right\} \tilde{\mathbf{X}}^{T} \tilde{\mathbf{H}}, \\
& =\left(\mathbf{X}^{T} \mathbf{X}\right)^{-1} \sum_{i=1}^{n}\left(\begin{array}{c}
\mathbf{H}_{i} \\
a_{i} \mathbf{H}_{i} \\
b_{i} \mathbf{H}_{i} \\
L_{i} \mathbf{H}_{i}
\end{array}\right) .
\end{aligned}
$$

Thus, $\log \mathbf{V}$ at a color point $(a, b, L)^{T}$ is estimated by

$$
\log \hat{\mathbf{V}}=\hat{\mathbf{M}}_{0}+a \hat{\mathbf{M}}_{1}+b \hat{\mathbf{M}}_{2}+L \hat{\mathbf{M}}_{3} .
$$

Then, the positive definiteness of $\hat{\mathbf{V}} \equiv \exp (\log \hat{\mathbf{V}})$ is obviously satisfied because eigenvalues of $\hat{\mathbf{V}}$ are positive.

\section{Numerical Experiments}

\subsection{Numerical experiments on DuPont dataset}

The proposed nonparametric formulas up to three bandwidths based on three normalization methods were applied to the DuPont training dataset. We have $9\left(=3^{2}\right)$ possible prediction formulas for positive definite matrices. The bandwidths were tuned by the leave-one-out cross validation according to the three metrics between observed and predicted matrices.

Table 3 compares $27\left(=3^{3}\right)$ models and lists tuned bandwidths. The first row separates the models according to the number of widths one, two and three corresponding to formulas (16), (22) and (26). The second row implies the $q$ th normalization methods with $q=1,2,3$ for each block. The 2 nd entry of the 4th row, 727.9 , gives the minimum Frobenius norm appearing in (16) with the optimal bandwidth based on the first normalization method

Table 3. Kernel estimation results.

\begin{tabular}{lcccccccccc}
\hline Number of bandwidths & \multicolumn{3}{c}{ One (14) } & \multicolumn{3}{c}{ Two $(20-21)$} & \multicolumn{3}{c}{ Three $(23-25)$} \\
\hline $\begin{array}{l}\text { Normalization methods: } q \\
\text { Metrics }\end{array}$ & $1(5)$ & $2(6)$ & $3(7)$ & $1(5)$ & $2(6)$ & $3(7)$ & $1(5)$ & $2(6)$ & $3(7)$ \\
Frobenius norm & 727.9 & 611.7 & 624.6 & 734.8 & 607.4 & 605.5 & 719.9 & $\mathbf{5 8 6 . 8}$ & 598.0 \\
Riemannian metric & 26.52 & 20.99 & 19.95 & 26.42 & 20.64 & 19.78 & 24.79 & 20.12 & $\mathbf{1 9 . 5 4}$ \\
KL divergence & 8.686 & 6.472 & 6.297 & 8.666 & 6.620 & 6.378 & 8.057 & 6.335 & $\mathbf{6 . 2 8 6}$ \\
Bandwidths & & & & & & & & & \\
$\gamma_{F}$ & 21.89 & 132.1 & 132.1 & 20.31 & 183.4 & 832.1 & 0.031 & 200.1 & 1532 \\
$\gamma_{R}$ & 25.59 & 1.771 & 29.80 & 13.47 & 1.563 & 28.63 & 0.031 & 2.250 & 36.78 \\
$\gamma_{K L}$ & 1.217 & 1.506 & 26.18 & 0.031 & 1.656 & 33.47 & 0.031 & 200.0 & 44.59 \\
$\delta_{F}$ & - & - & - & 26.91 & 1.750 & 20.75 & 16.78 & 1.250 & 256.08 \\
$\delta_{R}$ & - & - & - & 18.06 & 1.313 & 17.44 & 11.84 & 0.844 & 15.81 \\
$\delta_{K L}$ & - & - & - & 93.25 & 1.250 & 15.72 & 13.19 & 0.938 & 18.50 \\
$\eta_{F}$ & - & - & - & - & - & - & 16.47 & 1.813 & 21.00 \\
$\eta_{R}$ & - & - & - & - & - & - & 20.19 & 1.281 & 17.13 \\
$\eta_{K L}$ & - & - & - & - & - & - & 83.50 & 1.156 & 15.53 \\
\hline
\end{tabular}

Table 4. Evaluation of the estimated results by the three metrics.

\begin{tabular}{lcccc}
\hline & \multicolumn{3}{c}{ Estimation methods } \\
\cline { 2 - 4 } & \multicolumn{3}{c}{ Nonparametric } & Parametric \\
\cline { 2 - 4 } \cline { 2 - 4 } Evaluation metrics & Frobenius & Riemannian & Kullback-Leibler & Log normal \\
\hline Frobenius & $\mathbf{5 8 6 . 7 8}$ & 641.95 & 638.65 & 845.95 \\
Riemannian & 21.50 & $\mathbf{1 9 . 5 4}$ & 19.60 & 36.97 \\
Kullback-Leibler & 6.40 & 6.31 & $\mathbf{6 . 2 9}$ & 16.29 \\
\hline
\end{tabular}




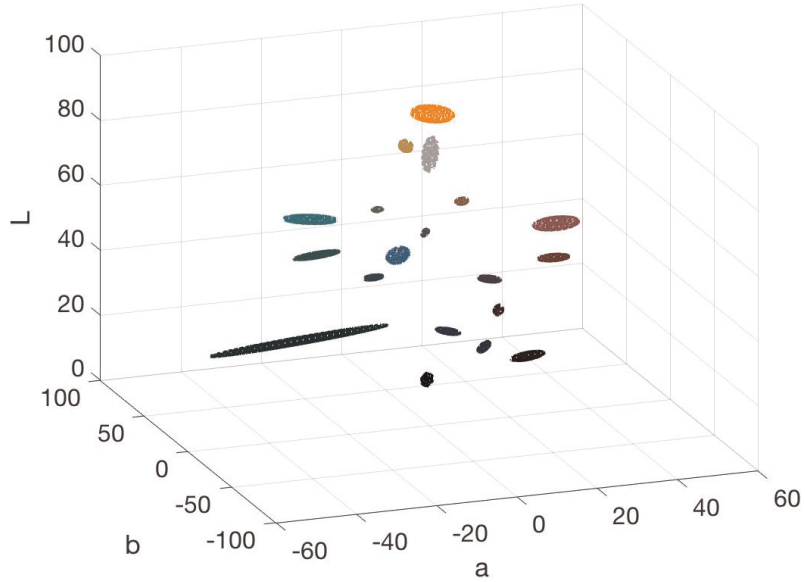

(a) True ellipsoids of 19 colors

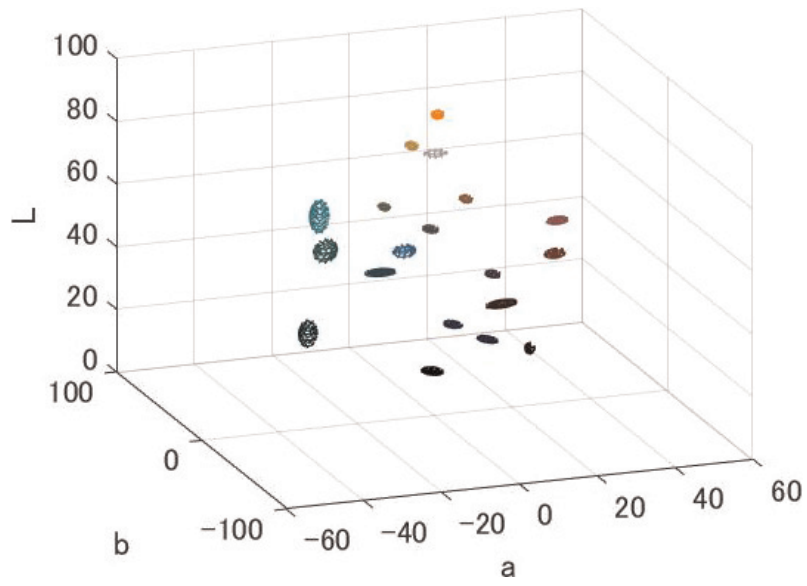

(c) Riemannian metric and 3rd normalization

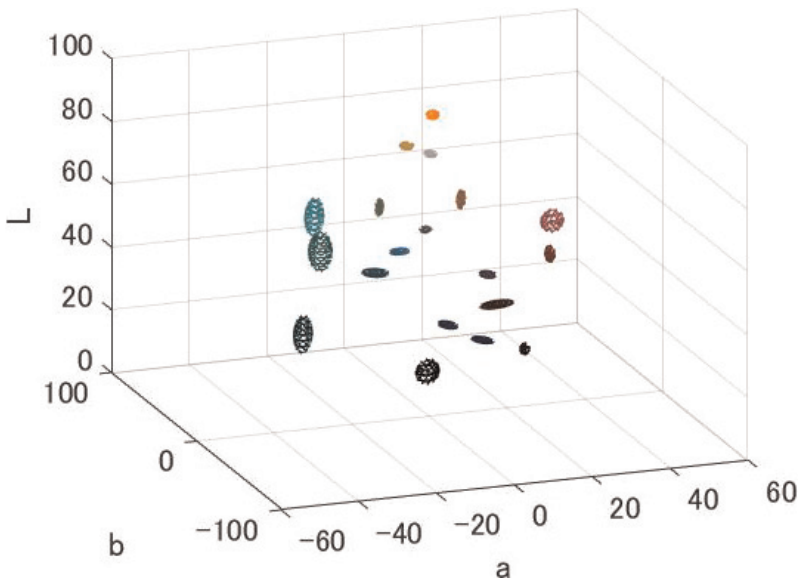

(b) Frobenius norm and 2nd normalization

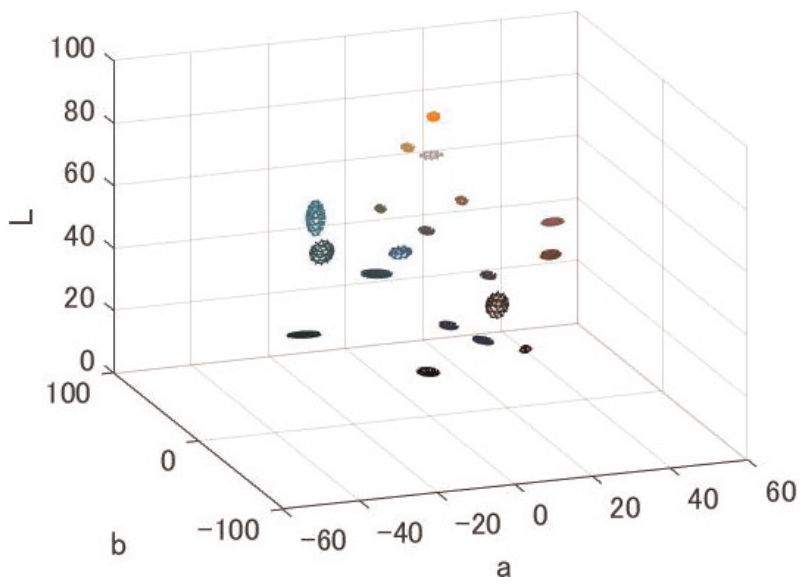

(d) KL divergence and 3rd normalization

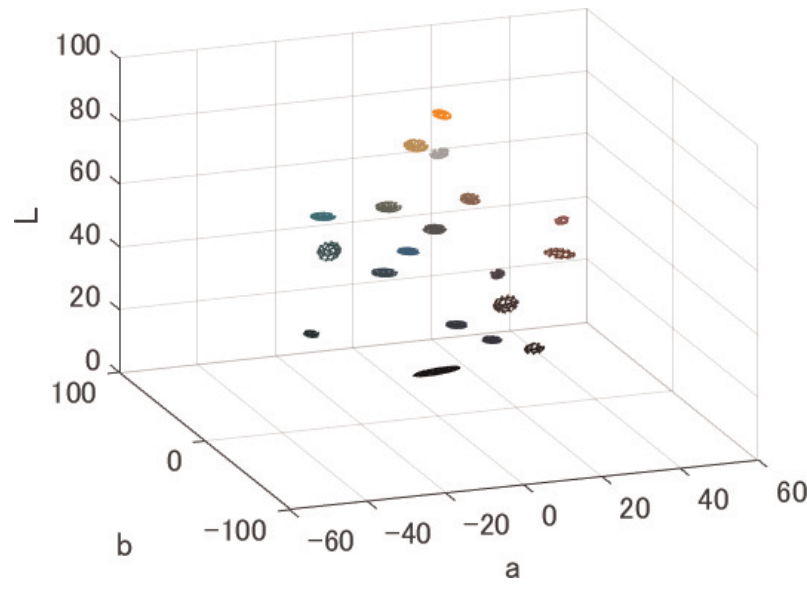

(e) Matrix log normal estimation

Fig. 2. True and estimated ellipsoids by the proposed methods. 
$q=1$. The 9 th entry in the 4 th row, 586.8 in bold face, gives the minimum Frobenius norm (26) in the same row. Similarly, numerals in bold face at the 5 th and 6 th rows mean that they are the minimum in the respective rows. We can see that the Riemannian metric and KL divergence chose the model with three bandwidths based on the third normalization method. The estimated bandwidths are tabulated from the 8 th to 16 th rows.

We can see from Table 3 that the models with three bandwidths had the best performance. Furthermore, the second and third normalization methods works well, and the difference between the second and the third normalizations is small. Table 4 compares four optimized models by the DuPont dataset. The second row shows the sum of Frobenius norms between 19 true and estimated matrices by each proposed method. The third and fourth rows are similarly derived by Rimannian and KL metrics. We use the three optimal kernel methods with three bandwidths found in Table 3 . The kernel methods based on Frobenius norm gives the minimum Frobenius norm, and so on. The accuracy of the log normal regression, however, is poor for the kernel methods.

\subsection{Estimated ellipsoids by the proposed methods}

Figure 2 depicts the true (a) and estimated ellipsoids by the kernel methods (b)-(d) and regression model (e). As shown, all the models failed to estimate the flat-shaped ellipsoid of "Dark bluish green". This may stem from the fact that this color is located at the edge of the 19 training colors. We can also see that the estimated ellipsoids in (e) by log normal regression are smaller than those by kernel methods in (b)-(d). The log normal regression, however, has an advantage because the linear estimation has a closed form of the solution. Also, the model can easily implement new explanatory variables such as second-order models.

\section{Conclusion}

It is known that each color has an ellipsoid region whose points are visually recognized as the same color. Currently, the DuPont dataset provides pairs of colors and ellipsoids in the CIE-Lab system. Of course, the shape of an ellipsoid is determined by a positive definite matrix. Therefore, in this paper, we have proposed the methods for estimating ellipsoids with a new color center in the Lab system.

First, we considered Gaussian kernel estimation of positive definite matrices with three bandwidths at most and three types of normalization methods of explanatory variables. We found that the third as well as the second normalization methods work well for estimation. The bandwidths were tuned by minimizing the three metrics, namely, Frobenius, Riemmanian, and KL. These three metrics showed similar performance for estimation. It is noted that this weighted-sum approach has a theoretical justification based on KL divergence.

Second, we used the matrix-valued log normal regression. This approach has a closed solution for regression coefficient matrices, and gives the ellipsoid of a new color easily. Unfortunately, it showed a poor performance with respect to kernel estimation. This may be improved by using additional explanatory variables derived by color features.

The paint shops at automotive factories currently need more than one month to adjust the painting conditions for synthesis of the same color. With our method, the color matching admitting tolerant ellipsoids could be easily tuned within one week because the painting condition of robot painters can be configured by applying tolerance ellipsoids. This would make it possible to mass-produce new colors with stable quality at factories around the world.

Furthermore, if human color sensitivity can be modeled, the synthesis of new colors could be developed by simulation. We plan to establish a fundamental technology enabling the efficient management of attractive new colors by developing more accurate prediction algorithms based on mathematical approaches such as support vector regression and other approximation techniques as well as on a database of colors and ellipsoids.

\section{Acknowledgments}

The authors are very grateful to the reviewers, whose constructive comments were very helpful in improving the paper. We would also like to express for our hearty thanks to the editor for kind handling of the paper. This research was supported by Mazda Motor Corporation and a Grant-in-Aid for Scientific Research (B) 15H02670. Takayoshi Nakamoto contributed equally to this paper. 


\section{Abbreviations}

CIE: Commission Internationale de l'Eclairage, KL: Kullback Leibler.

\section{Declarations}

Availability of data and material.

The datasets supporting the conclusions of this article are represented in Sec. 4.

\section{Competing Interests}

The authors declare that they have no competing interests.

\section{Author's Contributions}

All authors read and approved the final manuscript.

\section{References}

1. D. L. MacAdam, Color Measurement Theme and Variations, 2nd Revised edn. (Springer-Verlag, 1985).

2. J. P. Homann, Digital Color Management (Springer, 2007).

3. M. R. Luo, G. Cui and B. Rigg, The development of the cie 2000 colour-difference formula: Ciede2000. Color Res. 26(5) (2001) 340-350.
4. R. S. Berns and B. Hou, Rit-dupont supra threshold color - tolerance individual color - difference pair dataset, Color Res. 35(4) (2010) $274-283$.

5. M. Huang, H. Liu, G. Cui and M. R. Luo, Testing uniform colour spaces and colour - difference formulae using printed samples, Color Res. 37(5) (2012) 326-335.

6. S. Shen and R. S. Berns, Evaluating color difference equation performance incorporating visual uncertainty, Color Res. 34(5) (2009) 375-390.

7. M. Melgosa, E. Hita, A. J. Poza, D. H. Alman and R. S. Berns, Suprathreshold color-difference ellipsoid for surface colors, Color Res. 22(3) (1997) 148-155.

8. F. Hiai and D. Petz, Riemannian metrics on positive definite matrices related to means, Linear Algebra App. 430 (2009) 3105-3130.

9. J. Fan, N. E. Heckman and M. P. Wand, Local polynomial kernel regression for generalized linear models and quasi-likelihood functions, J. Am. Stat. Assoc. 90(429) (1995) 141-150.

10. W. S. Cleveland and S. J. Delvin, Locally weighted regression: An approach to regression analysis by local fitting, J. Am. Stat. Assoc. 83(403) (1988) 596-610.

11. Y. Yuan, H. Zhu, W. Lin and J. S. Marron, Local polynomial regression for symmetric positive definite matrices, Stat. Methodol. 74(4) (2012).

12. A. Schwartzman, Lognormal distributions and geometric averages of symmetric positive definite matrices, Int. Stat. Rev. 84(3) (2016) 456-486. 